\title{
Exploring the Structure of Regional Climate Scenarios by Combining Synoptic and Dynamic Guidance and GCM Output
}

\author{
JAMES S. RISBEY \\ School of Mathematical Sciences, Monash University, Clayton, Austalia \\ Peter J. LAmB \\ Cooperative Institute for Mesoscale Meteorological Studies, University of Oklahoma, Norman, Oklahoma \\ RON L. MiLleR \\ NASA Goddard Institute for Space Studies, New York, New York \\ Michael C. Morgan \\ Department of Atmospheric and Oceanic Sciences, University of Wisconsin-Madison, Madison, Wisconsin
}

GERARD H. RoE

Quaternary Research Center, University of Washington, Seattle, Washington

(Manuscript received 29 February 2000, in final form 4 September 2001)

\begin{abstract}
A set of regional climate scenarios is constructed for two study regions in North America using a combination of GCM output and synoptic-dynamical reasoning. The approach begins by describing the structure and components of a climate scenario and identifying the dynamical determinants of large-scale and regional climate. Expert judgement techniques are used to categorize the tendencies of these elements in response to increased greenhouse forcing in climate model studies. For many of the basic dynamical elements, tendencies are ambiguous, and changes in sign (magnitude, position) can usually be argued in either direction. A set of climate scenarios is produced for winter and summer, emphasizing the interrelationships among dynamical features, and adjusting GCM results on the basis of known deficiences in GCM simulations of the dynamical features. The scenarios are qualitative only, consistent with the level of precision afforded by the uncertainty in understanding of the dynamics, and in order to provide an outline of the reasoning and chain of contingencies on which the scenarios are based. The three winter scenarios outlined correspond roughly to a north-south displacement of the stationary wave pattern, to an increase in amplitude of the pattern, and to a shift in phase of the pattern. These scenarios illustrate that small changes in the dynamics can lead to large changes in regional climate in some regions, while other regions are apparently insensitive to some of the large changes in dynamics that can be plausibly hypothesized. The dynamics of summer regional climate changes are even more difficult to project, though thermodynamic considerations allow some more general conclusions to be reached in this season. Given present uncertainties it is difficult to constrain regional climate projections.
\end{abstract}

\section{Introduction}

The generation of regional climate scenarios for studying climate impacts has tended to follow several different methods, including use of climate models, empirical analogs, and use of simple offsets from the current climate (Pittock and Salinger 1981). The modeling method is by far dominant; a general circulation model

Corresponding author address: Dr. James S. Risbey, School of Mathematical Sciences, PO Box 28M, Monash University, Clayton, Vic 3800, Australia.
(GCM) is used to generate broadscale climate changes in response to anthropogenic greenhouse forcing. The output from the GCM is then typically "downscaled" from GCM gridbox scales to smaller scales using another numerical model (a mesoscale model), or via use of statistical relationships between the larger and smaller scales. The output generated on smaller scales is then used to drive impacts models.

From the outset it has been recognized that the output produced in this way may not necessarily be indicative of the climate changes that might occur in a region. Particularly for variables like precipitation, the projected 
sign may even be incorrect, and there is little reason to suppose that even ensemble runs within and across different GCMs would span the plausible space of possible regional precipitation changes. One method used to cope with this acknowledged uncertainty has been to employ "simple" climate change scenarios where precipitation and temperature are changed by increments spanning a range from decreases to increases (typically $\pm 20 \%$ for precipitation) (e.g., Gleick 1987; Lettenmaier and Gan 1990; Jeton et al. 1996). While this simple method succeeds in expanding the range of scenarios considered, it provides little information on the plausibility of the range, and thus has more in common with a sensitivity study than a climate scenario. The term climate scenario is generally used to refer to physically consistent (Smith and Kalkstein 1995) or plausible (Wigley et al. 1986) changes.

The "empirical" methods for generating greenhouse climate scenarios rely on the use of regional analogs in paleoclimatic reconstructions from warmer periods or on instrumental observations from warmer regions (Lamb 1987; Mearns et al. 1990; Giorgi and Mearns 1991). While capable of providing broad outlines of potential changes, analog approaches tend not to work well unless there is a large database of suitable analogs, as in the weather forecasting context. Analog databases tend to be more limited on climate timescales.

The numerical modeling methods are popular in part because they are deterministic, provide internally consistent scenarios, and seem to have more potential applicability to regions than the other approaches (Giorgi and Mearns 1991). However, the way in which modeling scenarios are generated at present tends to underutilize their full potential. This paper addresses one way in which that potential is not utilized. Present model scenarios tend to be just that-model scenarios. They generally have not been augmented much by human interpretation. In the early days of numerical weather prediction (NWP), the best forecasts were often those that combined model output with human interpretation. Such human-model forecasts were typically better than those produced by models or humans alone (Sanders 1979, 1986). NWP may have advanced to the point that this is no longer the case. Though the skill of present climate model greenhouse scenarios is difficult to assess, they are more closely akin to early NWP forecasts than contemporary NWP forecasts. When human interpretations were added to early NWP forecasts, the range of possible outcomes was often increased to more closely match the diversity of real outcomes [though cognitive biases can degrade forecasts as well, Nicholls (1999)]. That is because skilled analysts become aware of conditions under which the models tend to fail [e.g., under circumstances leading to explosive cyclogenesis, Bosart et al. (1995)] and can adjust the forecast accordingly. While regular feedback on model performance is not readily available for climate models, there are opportunities from simulation of past and present climates to develop a sense of model strengths and weaknesses. When climate scenarios are taken "verbatim" from models, such opportunities to augment the scenario with the modelers craft knowledge of the model are lost. Worse still, there may also be a number of unintended side effects of using model output verbatim in climate scenarios.

The (scaled) raw numerical output of GCMs is usually fed directly into a chain of subsidiary models-for instance, as when GCM output is used directly to drive regional climate models, output from which is then used to drive hydrological and agricultural models. This straight numerical "handoff" neglects dynamical nuances that may not be reflected in the raw model fields, but which may be discerned upon broader inspection of the model results. The details of the dynamics may often be important in determining local outcomes, and these details may actually be qualitatively captured in a model, if not in precise quantitative form with the correct spatial orientation. For example, a model may correctly convey a shift in a storm track, but may have the position slightly wrong. Alternatively, a modeler may recognize where a model systematically misplaces the midlatitude ENSO teleconnections. In both examples, this would be critical information for the climate of a particular region, but would not be correctly conveyed if the numerical output were taken literally in a numerical downscaling exercise.

Raw numerical output from climate models is less than optimal for the additional reason that it likely undersamples the range of plausible climate changes in a region. The physics in the model are not as rich as those in the real world. For example, natural variability is typically underestimated in model simulations (Barnett 1999). Whether omitted processes produce under- or oversampling of regional response depends on whether they tend to damp or amplify the system on balance. Regional climate responses will depend on the details of the patterns and strength of climate forcings (Schneider 1994). Depending on the spatial sensitivity of the system to these differences, one might have to perform many simulations with different plausible forcing patterns to properly sample different plausible response patterns. On a more optimistic note, Reader and Boer (1998) use GCM experiments to argue for a low spatial sensitivity, finding that "the pattern of climate response is determined, to first order, by the overall magnitude of the change in forcing rather than its detailed nature or structure."

Since climate model scenarios have hitherto been based on only a single (or few) forcing scenarios and only a single response (or small ensemble) to that forcing, we can expect only a subsampling from the "true" space of plausible responses. Since GCMs do embody something like our "best guesses" of the relevant physics for climate change, we might think of GCM climate scenarios as our best-guess scenarios. To be sure, a best guess is different from an attempt to explore the plau- 
sible space of scenario outcomes. A best-guess scenario may be more likely than other scenarios, but it does not encapsulate the full probability distribution of possible responses.

Another issue in forgoing an interpretation step in the use of climate model output for climate scenarios is that the assumptions on which the scenario are based are wholly intrinsic to the model. This is fine for the modelers, who understand the details of the model and model experiment parameters. However, the majority of users will not have much knowledge of the contingencies associated with the scenario.

We have argued that use of raw model output in generating climate scenarios tends to underutilize the dynamical information content implicit in the models, can introduce potential spatial biases in the scenarios, obscures assumptions, and constitutes only a best-guess view of climate change. In this work we attempt to address some of these issues by using expert judgement to interpret and augment the results from climate model runs in a similar spirit to the way in which weather forecasters interpret numerical weather model output. The experts in this case are climate modelers and dynamicists, along with synoptic meteorologists. The analysis was carried out in a series of communications between these groups, culminating in a workshop attended by all (Risbey et al. 1999). The synopticians begin by identifying features of the general circulation that control the synoptic climatology of a particular region. The climate modelers and dynamicists interpret changes in these features from the results of greenhouse climate model runs to produce large-scale climate scenarios. Finally, the synopticians use their knowledge of the synoptic climatology of the region to effectively downscale information from the large-scale climate scenarios.

For our first attempt at using expert judgement in this way, we have produced qualitative scenarios only, consistent with our assessment of the degree of precision afforded by existing uncertainties. In producing the qualitative scenarios we have attempted to make explicit the assumptions on which the scenarios are based, to draw dynamical insights from the models where possible, and to probe a broader range of plausible climate changes by articulating and defending alternative outcomes. An additional element of the scenario construction exercise is that we start by outlining key dynamical features that set regional climates and then systematically assess what is known about these features. By attempting to categorize uncertainties in all the features deemed relevant, we will therefore occasionally cover material of a more speculative nature.

Note that the emphasis in deconstructing regional climate scenarios here is on the role of dynamical processes. We do not ignore the role of thermodynamics, but focus mostly on the winter season when the dynamics are more prominent. We stress further that the process of deconstructing and constructing the scenarios is more important here than the end results. The process

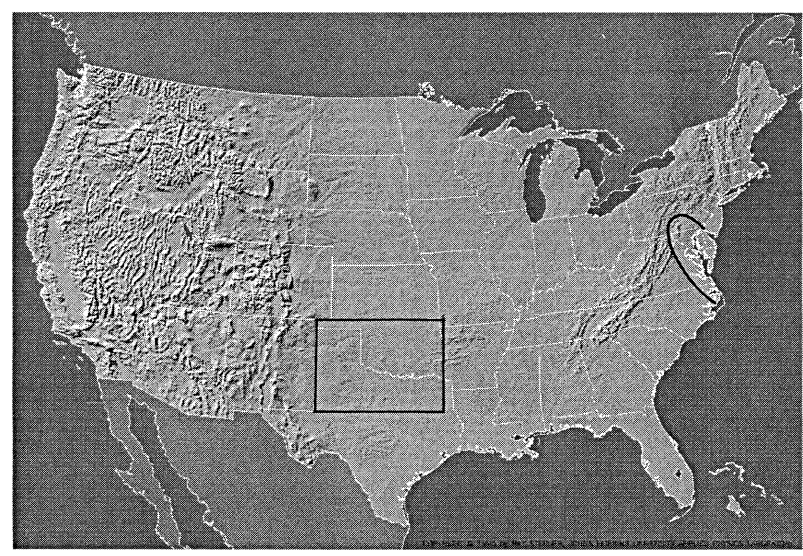

FIG. 1. Map showing the location of the two case study regions: the southern Great Plains, and the Chesapeake Bay.

is intended to highlight the elements, complexity, and dependencies underlying regional scenarios. We hope that this serves as a useful adjunct to GCM-based scenarios, but it is in no way intended for the method to compete with or replace such scenarios.

\section{Method}

Generation of the climate scenarios drew in particular (but not exclusively) on the results of model runs from the National Aeronautics and Space Administration (NASA) Goddard Institute for Space Studies (GISS; Russell et al. 1995, 2000) and the National Oceanic and Atmospheric Administration (NOAA) Geophysical Fluid Dynamics Laboratory (GFDL; Manabe et al. 1991) climate models. We selected two case study regions and carried out a number of preliminary analyses. The case study regions are the Chesapeake Bay region and the southern Great Plains region shown in Fig. 1. The regions were chosen to be large enough to have some economic and environmental significance, but small enough to be relatively homogeneous in their climatic characteristics and response. For brevity, we present mostly results from the Chesapeake Bay region here.

The method employed is "bottom-up" in that we began with each of the two case study regions. The synopticians for those regions characterized the current climate of their regions. They described the seasonal cycle of precipitation and temperature in the respective regions and the synoptic features that influence them. The seasonal cycle of precipitation in both these regions has contributions from winter and summer seasons. In the Chesapeake Bay the heaviest precipitation occurs during summer, although summer precipitation is more variable and less dependable than in winter. Wintertime precipitation in the region is associated with the passage of continental midlatitude storms and with those of subtropical origin moving northeastward along the Atlantic coast.

The synopticians also classified typical synoptic con- 


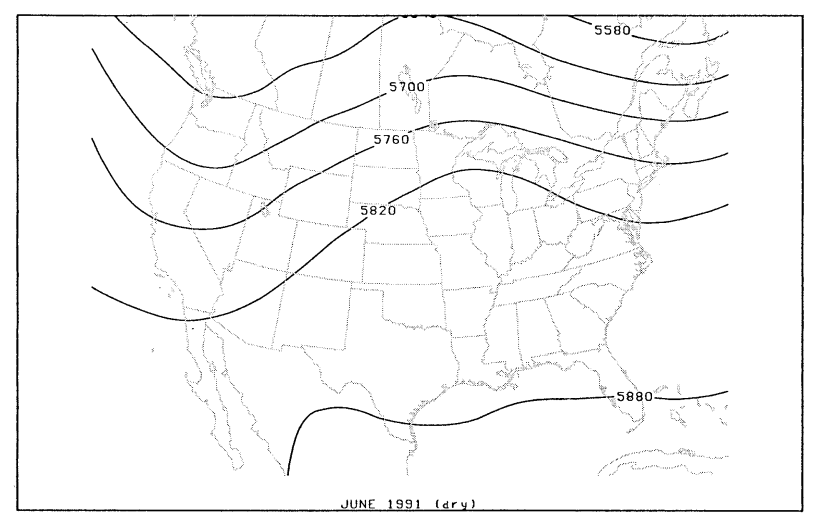

FIG. 2. The 500-hPa height field (m) for Jun 1991; typical of drier than normal summers in the Chesapeake Bay region. The precipitation in such months is about one quarter of the long-term average for these months.

figurations leading to anomalous precipitation in winter and summer in their region. In drier winters in the Chesapeake Bay region, the subtropical jet tends to be weaker and displaced farther to the south. The flow is more typically zonal over the eastern United States than in wetter winters, and is associated with fast moving, nonamplifying cyclones crossing the continent. In wetter winters, the subtropical jet is stronger and closer to the Chesapeake. Drier Chesapeake Bay summers are characterized by positive height anomolies over the continental United States [the characteristic "omega" $(\Omega)$ block exhibited in Fig. 2], while wetter summers are characterized by more zonal flow and a weak trough in the East Coast region (Fig. 3). Inspection of daily weather maps for wet summer months reveals periods in which vigorous upper-level troughs "cut off" over the Great Lakes and drift eastward over the study area.

The climate of North America and the Chesapeake Bay region undergoes coherent changes in association with Pacific-North America (PNA) variability (Wallace and Gutzler 1981). Positive phases of the PNA are associated with enhanced meridional flow patterns over North America in winter, while negative PNA patterns are associated with more zonal flow. Positive PNA anomalies are associated with below-normal temperatures in the Chesapeake region (Leathers et al. 1991). Precipitation correlations with the PNA cycle are weaker, though explosive cyclogenesis events on the East Coast are favored by positive PNA anomalies (Lackmann et al. 1997) and precipitation tends to be greater (Leathers et al. 1991).

The synopticians charged with doing the regional downscaling were asked the following question:

"In order to perform the downscaling for your region, what are the major large-scale and synoptic features for which you would like to have information in any new climate scenario? i.e. you have to produce a general description of the weather regime in your region and you are allowed information on only a small subset of syn-

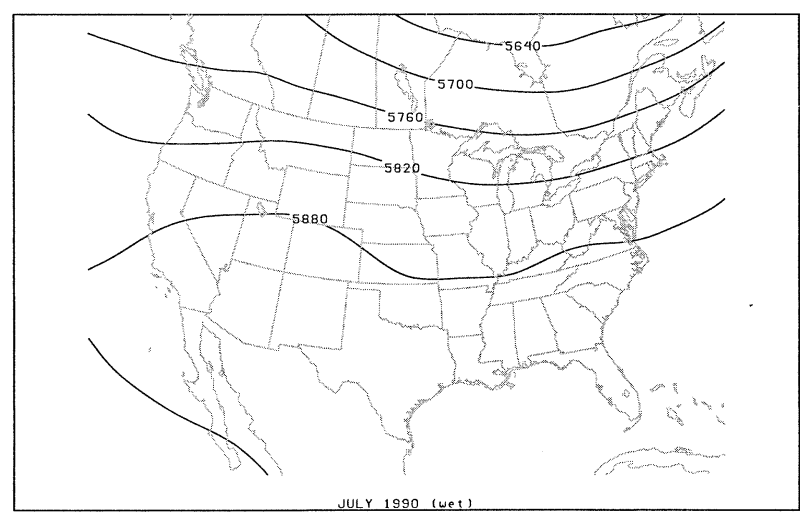

FIG. 3. The 500-hPa height field (m) for Jul 1990; typical of wetter than normal summers in the Chesapeake Bay region. The precipitation in such months is about twice the long-term average for these months.

optic features to do that-what is the subset of features that is most useful in allowing you to characterize any changes in local climate? These features will essentially comprise your 'toolkit' for the changed climate scenarios."

The downscaling method assumes that roughly the same set of large-scale dynamical features that control regional climate now will do so in the future. Increases in atmospheric moisture could change the efficiencies of dynamical transports and thereby change relationships between dynamical processes. However, such changes should be small for moderate $\mathrm{CO}_{2}$ perturbations, and thinking in terms of current dynamical constructs is a useful starting point. The features selected by the synopticians were as follows:

1) stationary wave field (over the Northern Hemisphere),

2) mean jet position (both subtropical and polar jet over North America),

3) storm tracks (over North America region at surface and upper troposphere),

4) mean temperatures and precipitation (over Chesapeake Bay region and southern Plains region),

5) El Niño-Southern Oscillation (ENSO)/La Niña,

6) PNA patterns.

For each of the first four features, information was requested for both winter and summer seasons. For the last two features a description of gross characteristics and changes only was requested. The climate modelers were asked to characterize the simulation of each of these features in current climate runs with their group's climate model, noting reasons for any deficiencies when known. They were also asked to describe how each of these features changes in enhanced $\mathrm{CO}_{2}$ simulations, to provide descriptions of why each of the changes occur, and of how well they thought the underlying mechanisms or reasoning is understood.

Responses from the climate modelers to the above questions provided the raw material for creation of the 
scenarios. The approach taken was to discuss changes for winter and summer separately. Because of the more coherent nature of the large-scale circulation in winter, we used the larger-scale features (stationary waves, jet streams) as the building blocks for the winter scenarios. In summer the situation is more complicated because of the general reduction in baroclinicity and in the coherence of the larger scales. Thus the approach taken for the summer scenarios was to focus more on the synoptic-scale characteristics of wet and dry summers in the regions and potential changes in these characteristics.

For winter we started our discussion of the large-scale features with the stationary wave field because it is so fundamental to regional climate (Lindzen 1994). While the stationary wave field steers the transients, the transients also contribute to the stationary waves. The discussion of tendencies for each separate feature cannot focus on that feature in isolation and must be more holistic in accounting for interactions between features. To follow the building block analogy, the building blocks all change into different shapes as soon as any one of them is put in place. Thus, we attempted to crossreference our discussion of each of the building block features wherever possible. Keeping track of such links is best done in a model, though many sensitivity experiments are required to tease out even a partial picture of the dynamics. Lacking a perfect model, there will always be some reliance on interpretation to construct that picture.

For each feature we classified the potential response to enhanced $\mathrm{CO}_{2}$ into one of the following categories as seemed appropriate to the level of understanding or predictive capability (bearing in mind that one can sometimes have high understanding, but still low predictive capability, as in a well-understood, but imperfectly sampled, chaotic atmosphere):

- Quantitative estimate: Can give the sign and magnitude of the expected change and the underlying reasoning.

- Definitive sign: Can give the sign of the expected change, explaining the reasoning and why changes of the opposite sign would not be expected (though they may occur-e.g., changes in temperature in high latitudes should exceed changes in low latitudes, though ocean circulation responses may confound that otherwise reasonable expectation).

- Ambiguous sign: Can give plausible arguments for the sign going either way.

- Speculative sign: Can give plausible arguments as to why the change might have one sign; cannot rule out changes of the opposite sign, but cannot give plausible arguments for how the opposite change would happen.

- Ignorance: Cannot give plausible arguments for how the sign might change in any direction.

The classification scheme provides an indication of tendencies, together with the underlying rational and levels of confidence. Throughout this exercise we assumed more or less an equivalent doubling of $\mathrm{CO}_{2}$ for the climate scenarios, though the precise perturbation is not so critical given that none of the responses were adjudicated to be in the "quantitative estimate" category. That is, the group felt that knowledge about potential changes in the above features was not good enough to make quantitative estimates for any of them.

The climate scenarios were synthesized from the component large-scale features, taking into account the plausibility of different tendencies in the features and dynamical consistency between the scenario elements. The classification scheme above provided a means to prioritize the tendencies selected in constructing the scenarios. For example, our "first-guess" scenario was constructed by selecting features whose $\mathrm{CO}_{2}$ response tended more to the "definitive sign" category.

\section{Results}

This section describes the results of the scenario construction exercise. We begin with a discussion of changes in low-frequency variability in response to enhanced $\mathrm{CO}_{2}$, and then discuss results for winter and summer circulation changes. A note on terminology is required at this stage. We will refer to both the subtropical and polar jets in describing the general circulation in this section. Synopticians usually maintain a distinction between these jets because they are often spatially distinct on daily synoptic maps. This distinction is sometimes lost on longer time averages. Dynamicists working with models frequently do not make this distinction and refer to a single zonal mean jet. In drawing implications from studies of a zonal mean jet for the synoptic jets, we will usually equate it with the subtropical jet, especially in regard to Hadley circulation studies. Note, however, that there is no requirement that the synoptic jets mirror the expectations for the zonal mean jet in specific locations, since the former can respond to stationary waves and local forcing. The nature of the relationship between the jets will be clear from the context in each case.

\section{a. Low-frequency variability}

While there are a variety of low-frequency modes that have been identified in the atmosphere and oceans (Lau et al. 1994; Trenberth 1997), our discussion considered only ENSO and the PNA, as these features were identified by the synoptic climatologists as more germane to the climate of their regions.

\section{ENSO AND PNA}

In the present climate, ENSO variability in the equatorial Pacific can influence climate in the extratropics through teleconnection patterns such as the PNA. This suggests that the midlatitude response to greenhouse forcing will depend in part upon forced changes to east- 


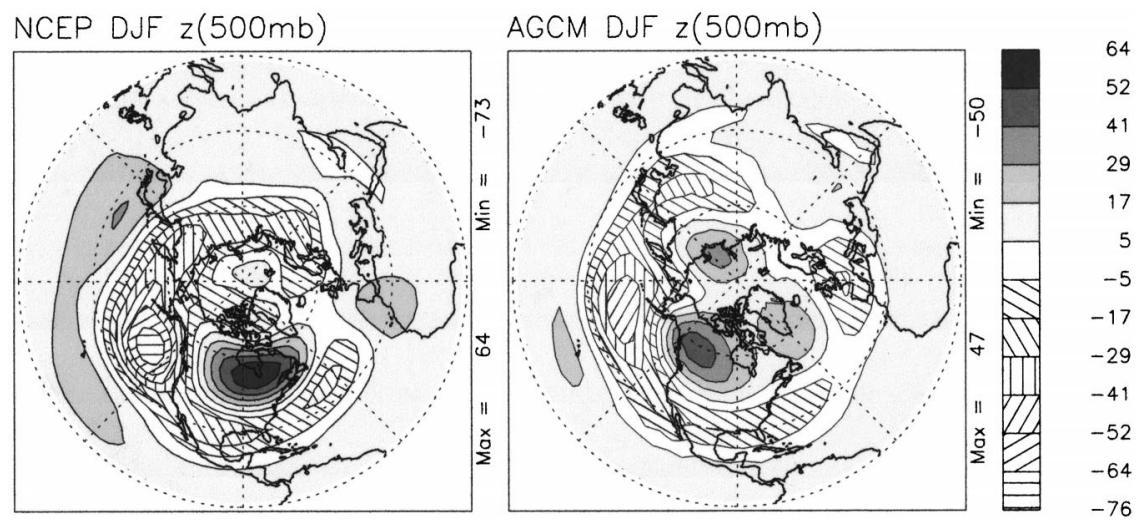

FIG. 4. Winter 500-hPa geopotential height anomalies (m) averaged over six ENSO events (1957/58, 1965/66, 1972/73, 1982/83, 1986/87, 1997/98) for observations (NCEP) and an AGCM forced by observed SST between 1951 and 1998. The AGCM estimate is derived from a 5-member ensemble. The AGCM is NASA GISS model B364M12.

ern equatorial Pacific climatology. However, expectations for changes to this region are at present controversial (Houghton et al. 2001). For example, some coupled GCMs suggest a warming in the eastern Pacific, associated with a reduction in the east-west temperature gradient and Walker circulation (e.g., Timmermann et al. 1999). However, it has been suggested that changes of the opposite sign are also possible (e.g., Cane et al. 1997; Cane 1998). The disagreement is not too surprising given that most coupled GCMs still run at resolutions that are too coarse to represent the dynamics of ENSO well, and exhibit "El Niño-like" behavior at best (Lau et al. 1992; Yukimoto et al. 2000). For better simulation of ENSO, higher meridional resolution is required to represent the dynamics of the ocean equatorial waveguide, and higher vertical resolution is required to capture the deepening of the eastern Pacific thermocline and its feedback on surface temperatures.

Many models [including the GISS model (see Fig. 4) and GFDL model (Lau and Nath 1996)] are able to capture the qualitative sense of the observed extratropical response to ENSO, which is manifest as the canonical PNA pattern. The PNA response to greenhouse forcing may depend on the ENSO response among other factors. The PNA could thus also increase or decrease in response to greenhouse forcing. In some GFDL model runs there is an increase in PNA index trends, consistent with the relative warming of the model eastern Pacific compared to the west. Tendencies toward more positive PNA values in response to greenhouse forcing also occur in the Hadley Centre (Carnell and Senior 1998) and Canadian Center for Climate Modelling and Analysis models (Boer et al. 2000), as in recent observations (Corti et al. 1999). There are also reasons to project more negative PNA values. A weakened pole-to-equator temperature gradient (Gitelman et al. 1999) could lead to reduced topographic forcing, a more zonal stationary wave pattern, and more negative PNA anomalies.

The task of characterizing the PNA response is dif- ficult as there is still considerable debate over the nature and location of the mechanisms that excite the PNA teleconnection pattern. On timescales of days to weeks, Dole and Black (1990) argue that internal atmospheric processes (e.g., instability of the climatological mean flow) excite the PNA. Other studies focus on the role of boundary layer anomalies [in particular, tropical SSTs (ENSO), e.g., Horel and Wallace (1981)]. Both mean flow instability and SST forcing processes are invoked above in discussing potential PNA changes. Any net change in the PNA pattern would depend on which of the two processes were more influential. In summary, plausible arguments can be given to support changes in ENSO and PNA of either direction, and the response is best classified as "ambiguous sign."

\section{b. Winter}

\section{1) Stationary WAVES}

Atmospheric stationary waves are generated by largescale zonal asymmetries in climate forcing. Such forcing may be orographic (deflection of the flow over mountains), diabatic heating (e.g., land-sea thermal contrast, latent heating), or the result of convergence of heat and momentum fluxes due to transient eddies (e.g., Hoskins and Valdes 1990). The structure of the zonal mean jet is determined by the supply of angular momentum by the Hadley cell, the latitudinal gradients in radiative forcing, and the transport of heat and momentum by transient eddies. This structure creates the refractive index through which the stationary waves propagate (e.g., Nigam and Lindzen 1989), and the strength and position of the zonal mean jet at low levels determines the amount of forcing felt by the atmosphere (Nigam and Lindzen 1989; Held and Ting 1990). While GCM greenhouse forcing integrations consistently predict a weakening of zonal mean low-level temperature gradients (Gitelman et al. 1997), model studies differ as to even 
the direction of change of the Hadley cell intensity and extent (Collins and Karoly 1996; Rind 1998), which is acutely sensitive to small changes in the location and concentration of zonal mean tropical heating (Lindzen and Hou 1988; Hou and Lindzen 1992).

The stationary wave response (amplitude and phase) at any given location is a combination of the response to local forcing and the downstream propagation of waves forced remotely. We will discuss contributions from local and remote forcings separately.

Large changes in local forcing are required to produce large changes in the phase locally. Since doubled $\mathrm{CO}_{2}$ worlds do not involve such large changes, the main consideration for local forcing will be changes in amplitude of the stationary waves. One of the more robust results from greenhouse forcing experiments with climate models is a wintertime warming of mid- and highlatitude continents, associated with a reduction in snow cover. This markedly reduces the wintertime land-sea temperature contrast over the east coast of North America. This has three effects. First, the land-sea thermal contrast drives a low-level anticyclonic circulation over land in winter, which would consequently be reduced. Second, because air coming off the continent is now much warmer, the sensible and latent heating in the western Atlantic is greatly reduced. This heating is an important source for developing winter storms, and so the expected reduction in land-sea thermal contrast along the East Coast should lead to fewer (or weaker) storms developing along the east coast in the wintertime (Hall et al. 1994; Carnell and Senior 1998). And third, decreased continental snow cover will reduce the occurrence of extreme cold air outbreaks responsible for some of the more frigid winter conditions on the East Coast.

Wintertime stationary waves in the North American region would also be affected by any changes in strength of the prevailing westerly airflow across the continent. Reduced strength in the continental westerlies would reduce the amplitude of orographic forcing of the stationary wave pattern in the lee of the Rocky Mountains. This would result in more zonal flow across the continent. In contrast, Held (1993) has suggested that with a smaller temperature gradient, air must be brought in from farther away to balance the temperature changes associated with the vertical motion. This could be achieved by a more zonally asymmetric flow. However, this does not seem to occur in summer when the meridional temperature gradient is weaker. Thus, assuming a more zonal response, storms would track more nearly zonally eastward, reducing the incidence of storms that "dip down" across the continent and pick up moisture from the Gulf of Mexico region. Caveats to this linear thinking are that the orographic response may be saturated, or that the centers of action of the response may shift as the forcing changes (e.g., Trenberth and Chen 1988). However, we feel that the size of predicted changes to the time mean flow are such that "perturbation-type" thinking may be applied.

While local forcings can change the amplitude of the stationary wave pattern locally in the kinds of ways described, they are less efficient at changing the phase of the pattern locally. However, a small change in the atmospheric structure (refractive index) can have a large impact on the remotely forced stationary waves. For instance, it is readily shown using a barotropic model (e.g., Held 1983) that changes of a few meters per second in equivalent barotropic jet strength over the Tibetan Plateau can easily lead to phase changes of order $90^{\circ}$ in the stationary wave pattern over the North American continent. Many studies indicate that the atmospheric response to the Tibetan Plateau contributes to the wintertime upper-tropospheric high pressure ridge over western North America. The amplitude of the stationary wave caused by the Tibetan Plateau is sensitive to the position of the zonal mean jet in the region (Nigam and Lindzen 1989). An equatorward displacement of the jet leads to an increase in the amplitude of the induced pattern.

Other remote forcings of the stationary wave pattern include the contributions from latent heat release in storm tracks. While weaker jets may in general retard the growth of transient eddies, the increase in evaporation characteristic of a warmer climate may compensate by leading to larger latent heat release within storms. This could change the magnitude of heating within the storm tracks, further compounding changes in heating patterns due to shifts in locations of the tracks themselves.

This is by no means a complete survey of potential changes in stationary wave response, though we have tried to cover some of the major forcings of the pattern. Expectations about local forcings of the wintertime stationary wave pattern over North America include a reduction in land-sea thermal contrast and increased zonality of the pattern. There are good reasons to expect the reduction in land-sea contrast and this can be labeled as "definitive sign." The contribution to increased zonality is partly "ambiguous" as it relies on projections of a decrease in low-level zonal mean jet strength and may be counteracted by the need for increased northsouth advection in the lee of the mountains. The contributions to stationary wave changes over North America from remote sources are even more difficult to project due to the acute sensitivity of the downstream patterns to atmospheric structure, which is highly dependent on various model details and parameterizations in climate models (e.g., Risbey and Stone 1996). For example, the low-level winds, which do much of the topographic forcing of the stationary wave pattern, are not well simulated in most GCMs. Thus a model's remotely forced stationary wave response is largely unknown and characterized by a fair degree of ignorance at this stage. For example, Siegmund (1992) and Stephenson and Held (1993) come to differing conclusions 
as to the source of changes seen in different GCM simulations.

\section{2) JET STREAMS AND STORM TRACKS}

We led with a discussion of the stationary wave response, since the stationary waves determine the locations of the storm tracks. However, the storms also act to force the stationary waves, and neither can be viewed as external to the other. In this section we discuss expectations for the jet stream and storm track responses over North America.

GCMs disagree in their predictions of changes in the jet streams. Some climate models (e.g., GISS) project a decrease in the strength of the wintertime subtropical and polar jets over North America in response to greenhouse forcing. The jets apparently weaken due to the general reduction in baroclinicity in association with reduced tropospheric temperature gradients between equator and pole and between land and sea. In other models (e.g., GFDL R30 coupled model) the polar jet increases in strength over the North American region in association with more local increases in baroclinicity. This could occur, for example, in a scenario where snow melts over the United States but not over Canada, thereby enhancing the meridional temperature gradient locally. The subtropical jet also increases in strength in the GFDL model, suggesting that the model is responding to the increase in meridional temperature gradients in the upper troposphere brought about by widespread upper-tropospheric warming contrasted with the cooling of the lower stratosphere at the same height poleward of the jet. Some studies suggest that the subtropical jet is more closely tied to upper-(than lower) tropospheric baroclinicity (Palmen and Newton 1969; Morgan and Neilsen-Gammon 1998).

Though the displacements of the jet streams are hard to predict, there is a general expectation from models that the winter polar jet may shift poleward in response to greenhouse forcing, reflecting local changes to the region of maximum surface temperature gradients. Specifically, the maximum in warming in high latitudes during winter reduces the static stability there, shifting the region of greatest baroclinicity poleward (Held 1993). Displacement of the winter subtropical jet over North America may depend more on changes in uppertropospheric circulation. Since changes in the Hadley cell intensity/extent can be argued in either direction, so too can changes in the displacement of the subtropical jet.

The wintertime North Pacific and North AmericaAtlantic storm tracks are expected to shift poleward in association with the poleward shift in the jet streams and the regions of greatest baroclinic instability. The storm tracks might also become more zonal in orientation in association with an increase in zonality of the stationary wave and jet stream patterns. However, because transient eddies are coherent with height through the troposphere it is not clear whether they will respond in general to the reduction in temperature gradients at the surface or the expected intensification of the gradients aloft (Held 1993). The widely expected increase in moisture in a warmer atmosphere will also affect midlatitude eddies. Increases in evaporation and latent heat release (and/or changes in local baroclinicity) would lead to stronger eddies and enhanced downstream development in the storm tracks (Hall et al. 1994). Enhanced downstream development may also occur because a weakened jet means slower advection of eddies downstream out of the baroclinic zone. By contrast with the above, a more moist atmosphere would need weaker or fewer eddies to accomplish the same energy transport.

The above are general arguments on the scale of the North American continent. For more specific regions, changes in storm intensity and location will also be tied to changes in salient synoptic features. On the West Coast for instance, a strengthening of the Aleutian low could lead to more intense eddies on the West Coast. We have already mentioned that the reduction in temperature contrast along the East Coast is expected to weaken those storms that intensify in that region.

In summary, with some confidence, we expect the wintertime polar jets and midlatitude storm tracks to move poleward. Though there is some expectation of a weakening in intensity of the jets and midlatitude storms, there are plausible arguments to support a strengthening as well. A more robust expectation is that storms will intensify downstream in the storm tracks. Changes in the subtropical jet are even harder to characterize and plausible arguments can be given for changes in intensity and migration in either direction. These depend on the precise nature of the changes in heating and Hadley circulation in the Tropics and on the sensitivity of the jet to these changes.

\section{3) SYNTHESIS}

The task of synthesizing possible trends in a variety of large-scale features to produce a climate scenario is nontrivial. The overall response of the system will be a dynamic superposition of changes in the underlying features. Use of a model is generally the best way to ensure dynamical consistency. In this case we are using models to provide guidance on the kinds of combinations of scenario elements that accord with dynamical reasoning. However, one might not want to use models exclusively, since the models have difficulty representing some of the most crucial elements of the scenarios (such as the stationary wave response) reliably. For example, the current suite of GCMs have substantial differences in storm track intensity and location (e.g., Kageyama et al. 1999). This is all the more significant in that heating and convergence of dynamic fluxes within the storm tracks act in part to force the stationary waves (e.g., Wang and Ting 1999). The scenarios provided by this synthesis are hypothetical, though so are present 
TABLE 1. Possible response of selected large-scale climate system features to increases in greenhouse gases. "Trend" refers to an expectation of the most likely trend. The terms categorizing the sign of the trend are defined in the text.

\begin{tabular}{|c|c|c|c|}
\hline Feature & Trend & Sign category & Comments \\
\hline ENSO/La Niña & $?$ & Ambiguous & Low confidence in models \\
\hline PNA & $?$ & Ambiguous & Low confidence in models \\
\hline Arctic Oscillation & $?$ & Speculative & \\
\hline $\mathrm{N}-\mathrm{S}$ temperature gradient & Decrease & Definitive/ambiguous & Ocean may confound \\
\hline Hadley cell strength & $?$ & Speculative/ambiguous & \\
\hline Hadley cell extent & $?$ & Speculative/ambiguous & \\
\hline Polar jet strength & Weaker & Definitive/ambiguous & Reduction in N-S temperature gradient \\
\hline Polar jet position & Poleward & Definitive/ambiguous & Max baroclinic zone moves $\mathrm{N}$ \\
\hline Subtropical jet strength & Weaker & Ambiguous & \\
\hline Subtropical jet position & Equatorward & Ambiguous & \\
\hline Storm track position & Poleward & Ambiguous & \\
\hline Downstream intensification & Increase & Definitive/ambiguous & More latent heat release \\
\hline Eddy intensity & Weaker & Ambiguous & \\
\hline \multicolumn{4}{|l|}{ Stationary wave sources } \\
\hline — land-sea contrast & Reduced & Definitive & Fewer east coast storms \\
\hline — topographic response & More zonality & Ambiguous & Assuming weaker jets \\
\hline - remote sources & $?$ & Ignorance & Implications for phase \\
\hline Omega blocks & $?$ & Speculative & Important for drought \\
\hline
\end{tabular}

model-only regional scenarios. Consistency cannot be guaranteed in the scenarios, but is produced by ensuring that the trends in dynamical building blocks are combined in ways that make at least minimal sense.

The basic elements of the scenarios are summarized in Table 1, which shows the projected trends in each of the major features discussed above. In each case we have labeled the degree of confidence afforded each trend estimate according to the sign categorizations defined in section 2. In many cases, plausible arguments can be advanced for projecting a change in sign in either direction (ambiguous sign), depending on uncertainties in the sensitivity of the feature in question and on trends in other features on which it depends.

In synthesizing scenarios from these elements we started first with key features for which the projected sign is more definitive than ambiguous. This we labeled our first-guess scenario. We then produced a secondguess scenario by choosing plausible changes of the opposite sign in important features where the projected sign was categorized as ambiguous. Finally, we tried to produce a "radical change" winter scenario where we deliberately selected tendencies likely to produce more extreme regional scenarios. This scenario was an attempt to probe the scenario space further, to see how easily such scenarios could be generated, and to ask whether more extreme changes could be ruled out on dynamical grounds. Each of these scenarios will now be described in turn.

\section{4) First-Guess SCENARIO}

The first-guess scenario assumes a general reduction in meridional temperature gradient consistent with more rapid warming in polar regions than equatorial regions, along with more rapid warming over land than ocean regions. From this, one plausible scenario is for the following:
- a general reduction in baroclinicity at low and midlevels in midlatitudes,

- a weakening of the polar jet stream,

- a reduction in orographic forcing and increase in the zonality of the stationary wave pattern,

- a poleward shift of the zone of maximum baroclinic instability and poleward displacement of the polar jet,

- weaker land/sea temperature contrasts along the East Coast and weaker East Coast storms.

Assuming in addition a reduction in Hadley cell extent, this scenario also entails

- a weaker zonal mean (subtropical) jet displaced toward the equator.

This set of changes is similar to, and broadly consistent with, the results of GCM enhanced $\mathrm{CO}_{2}$ experiments. We provide a schematic representation of the changes in the jet stream in Fig. 5. Over central and eastern North America the polar jet axis has moved farther north into Canada in this scenario, while the subtropical jet has moved equatorward. This means that the two jets no longer merge as strongly in the vicinity of the East Coast, as in the current climate (note however that the jets must merge to some degree over the Atlantic; for there to be two distinct jets over the Atlantic, there would need to be two distinct maxima in meridional temperature gradients over the Atlantic, which seems unlikely). This will lead to weaker jets in this region in general.

From these broad changes in large-scale dynamical features, the synopticians were asked to provide an effective downscaling by arguing how the climates of their region would respond to these changes. For the Chesapeake Bay region the winters would become substantially dryer. This is because there would be both fewer and weaker East Coast storms. There would be fewer storms of this type as the more zonal stationary wave 


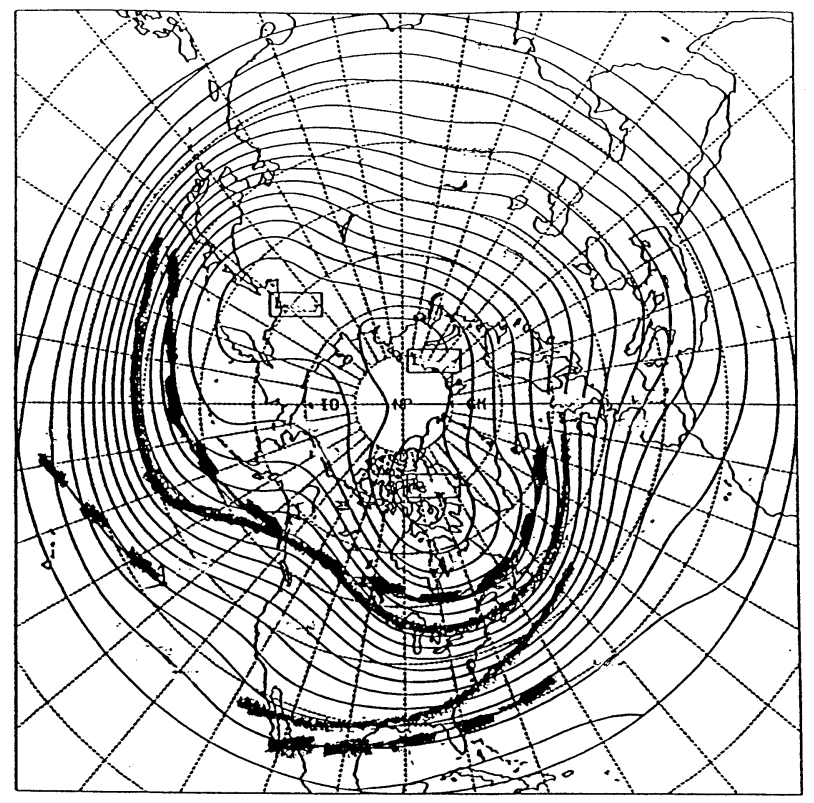

FIG. 5. Schematic representation of the wintertime polar and subtropical jet streams in the North American region. The thick solid lines show the approximate winter mean locations of the jets for the current climate and the thick dashed lines show locations for the firstguess scenario. The thin solid lines provide a representation of the stationary wave field for the current climate via the winter mean geopotential height $(\mathrm{m})$ at $500 \mathrm{hPa}$.

pattern would lead to more zonal flow of storm systems across the continent, with fewer storms tracking up the East Coast entraining moisture from the Gulf of Mexico region. Further, such storms as did move in this manner would generally be weaker due to the reduction in baroclinicity in the East Coast region. The Chesapeake Bay would also warm in this scenario due to the general increase in longwave radiation and because there would be fewer incursions of cold air from Canada with a more zonal flow pattern. For the southern Great Plains region, there would also be less precipitation and warmer temperatures in this scenario. This is because there would be a reduction in lee cyclogenesis from the Rocky Mountains and fewer storms in the Great Plains region from this source. A more equatorward subtropical jet would also imply fewer storms in the southern Great Plains.

\section{5) SECOND-GUESS SCENARIO}

For the second-guess scenario we focused more on the Tropics as a potential driver of regional climate changes. In this case we assumed a warmer Tropics, particularly in the eastern Pacific Ocean. This might come about for instance via increases in the frequency and/or intensity of ENSO events such as observed (Trenberth and Hoar 1996) or simulated in some climate model greenhouse experiments (Timmermann et al. 1999).

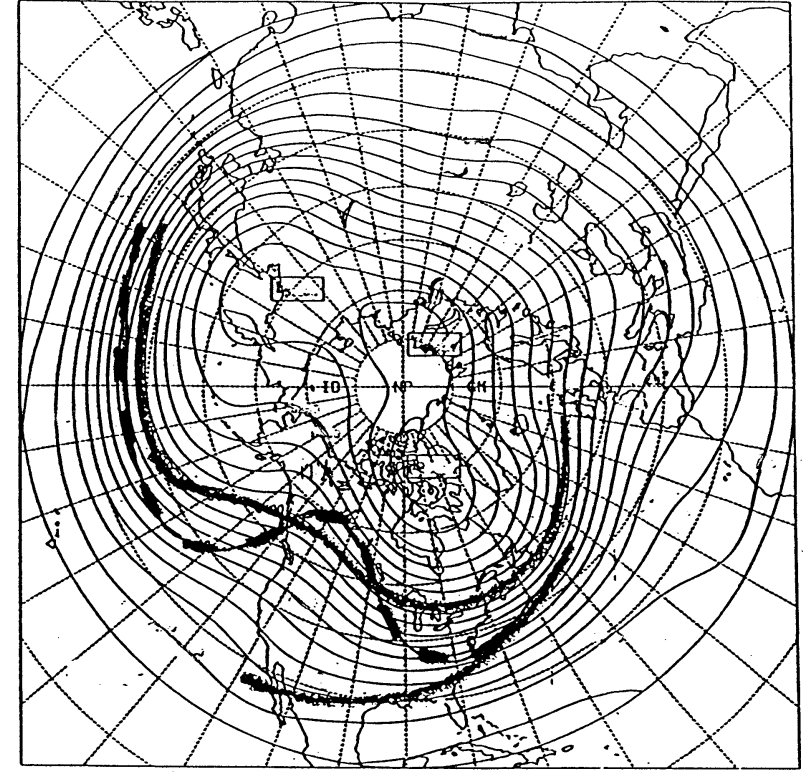

FIG. 6. As in Fig. 5, but for the second-guess scenario.

An enhanced warming in the eastern Pacific would imply the following:

- a weaker Walker circulation due to the reduction in east-west temperature gradients,

- a displacement of the intertropical convergence zone (ITCZ) closer to the equator,

- and a strengthening of the Hadley cell and subtropical jet, as observed during present-day ENSO events.

These changes in tropical circulation could induce changes in extratropical circulation. Figure 4 shows composite maps of the extratropical circulation during ENSO years in observations and the GISS climate model forced with observed SSTs. The extratropical tendencies associated with these ENSO events show the canonical seasonal timescale PNA pattern with a negative height anomaly over the Gulf of Alaska, a positive anomaly over the northern Rockies, and a negative one over the southeastern United States. Thus in this scenario we projected an enhanced (positive) PNA pattern in association with the eastern Pacific warming. The polar jet stream would change in association with the enhanced PNA pattern by increasing its zonal asymmetry as depicted in Fig. 6.

In this second-guess scenario, the winter climate of the Chesapeake Bay region would become wetter and stormier in conjunction with the enhanced PNA pattern and southward displacement of the polar jet over eastern North America. This is consistent with the PNA-Chesapeake climate correlations reported in section 2 . The studies cited there on PNA associations also suggest a reduction in temperature in the Chesapeake region associated with enhanced entrainment of cold air from Canada in this jet stream configuration. This cooling 
might be counteracted somewhat by the greenhouseinduced increase in radiative forcing. For the Great Plains region the more active subtropical jet would produce more storms in the region and hence a wetter winter climate. The wet winter projection for this enhanced ENSO scenario is consistent with the tendency for current ENSO years to favor wetter conditions in the Great Plains region (e.g., Ropelewski and Halpert 1986; Montroy et al. 1998). The increase in cloudiness associated with the extra storm activity in the region would contribute to a reduction in diurnal cycle and an overall warming in the region.

\section{6) RAdical Change SCENARio}

For a third winter scenario we set about trying to hypothesize a more radically different scenario that was still plausible and dynamically consistent. Expert elicitation methods often ask experts to describe conditions that might produce more extreme results as a way to probe confidence in the tails of expected distributions (Spetzler and von Holstein 1975). That is, one attempts to defend the assigning of low probabilities to certain scenario outcomes by describing conditions under which they could or could not occur. Radical changes in any midlatitude region could be brought about by shifts in the stationary wave pattern and associated jet streams and storm tracks. In principle, one of the best ways to change the stationary wave pattern and storm tracks is to induce phase shifts in the pattern by changing the refractive index of the atmosphere downstream from source regions. As discussed earlier, a small change in speed of the jet over the Tibetan Plateau can, in principle, induce a $90^{\circ}$ phase change in stationary wave pattern over North America. If such a phase change were imposed on that pattern this would strongly reduce the climatological mean ridge over the west coast of North America as indicated in Fig. 7. This would have major implications for the climate of the west coast region. However, the trough over the eastern part of North America has not been moved downstream in this scenario, and the stationary waves and jet structure in this region are largely unchanged as depicted.

The reason the trough over eastern North America has not been moved downstream in this scenario is that it is largely anchored in place by the effect of topographic forcing over the Rocky Mountains. The trough sets up in the lee of the Rockies irrespective of upstream changes in refractive index. It is difficult to imagine shifting that trough around much through upstream changes in jet strength, and it may be a fairly robust feature of the winter circulation in that regard. Substantially eliminating the trough by changing the speed of the upstream jet would require very large changes in jet speed, perhaps on the order of 50\% reductions or so. Since there have been no suggestions of such large changes in the jet in response to greenhouse forcing, we did not consider it to be a plausible option in this

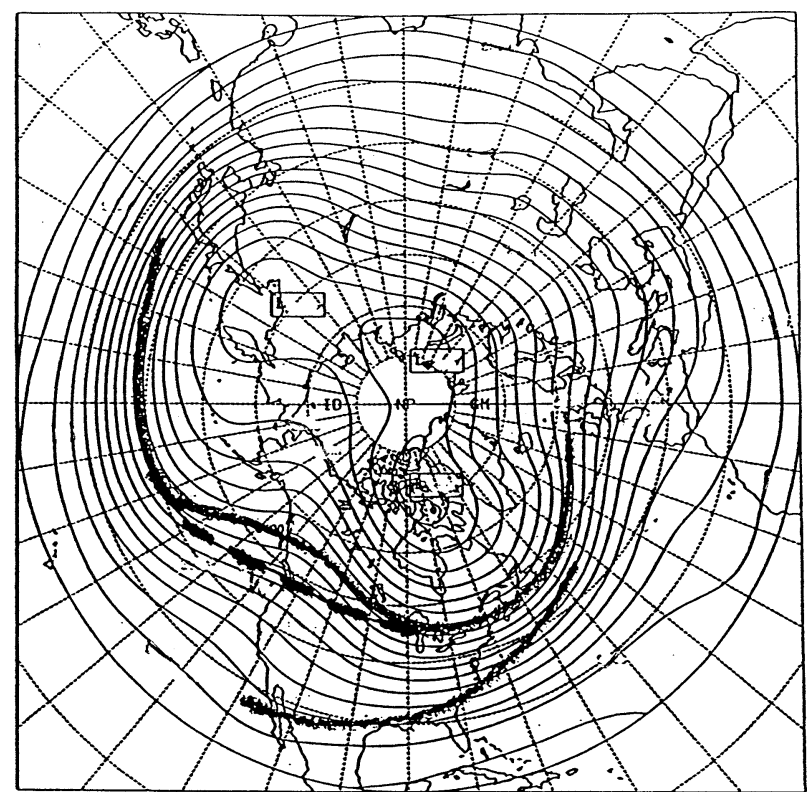

FIG. 7. As in Fig. 5, but for the radical change scenario.

scenario. To shift this trough out of position would require that the balance between topographic forcing and heating for the stationary wave pattern in this region shift heavily toward heating. In general, the relative importance of topographic forcing and heating depends on the strength of the low-level winds. The faster the low-level winds the more topographic forcing and the less time for heating to influence the pattern. In summer the northern jet streams are about $50 \%$ weaker than they are in winter in association with the large reduction in meridional temperature gradient then. In summer at upper-tropospheric levels there is still a topographic signature from the summer jet streams (a weak trough and storm track), but at lower levels the stationary wave pattern is dominated by the heating pattern. The seasonal change in meridional temperature gradient is large compared to the projected reductions from greenhouse forcing [the seasonal change in high-latitude temperatures is about $25 \mathrm{~K}$ (tropical temperature changes with season are small) Peixoto and Oort (1992), whereas projected changes for the same season for $\mathrm{CO}_{2}$ doubling in high latitudes are typically much less than half that], and so the balance of winter forcing would most likely stay with topography in any greenhouse scenario. Thus we consider the winter eastern North America trough in the stationary wave pattern to be a fairly robust feature, unless one can devise plausible ways to imagine very large changes in jet speeds.

Ironically, our attempt to devise a more radical change scenario by hypothesizing large changes in the phase of the stationary wave pattern ended up producing relatively small changes in the regions most affecting the localities we chose to examine. If anything, we were led to conclude that the winter climate of the East Coast 
is somewhat insensitive to processes that might change the phase of the stationary wave pattern. To be sure, we did not explore the range of other possible avenues via which the winter climates of this region may be radically changed, and we cannot rule out large changes. However, our radical change scenario would probably not result in large changes to temperature and precipitation in our regions of interest.

\section{c. Summer}

In summer, there is not such a clear relationship between large-scale dynamical features and the local climate as there is in the winter, because of the much reduced meridional temperature gradient and atmospheric baroclinicity. There is a weaker stationary wave pattern and local climates are much more dependent on local surface and boundary layer processes. Simulation of regional precipitation requires knowledge of surface energy budgets, evapotranspiration over the continents, low-level winds, and moisture advection, for instance. These processes occur on mesoscales that are not well resolved and simulated in GCMs. Indeed, GCMs tend to disagree on the projected response of precipitation to greenhouse forcing in specific regions in summer (Grotch and MacCracken 1991; Boyle 1998). The approach taken for the winter scenarios of assessing changes in large-scale dynamical features from GCM simulations is not nearly as applicable for summer. However, there are general thermodynamic arguments gleened from climate model studies that can provide some assistance.

For temperature and atmospheric moisture there is a general expectation of increases in the longer run in all seasons associated with the persistent greenhouse forcing. General increases in temperature and humidity lead to increasing incidences of "heat" related impacts (Delworth et al. 1999). There are also general conclusions that can be argued for the hydrological cycle over continental areas (wet regions tend to get wetter, dry regions tend to get drier) that are well grounded from thermodynamic considerations (e.g., Hansen et al. 1989). Further, a variety of studies have argued for general increases in precipitation intensity based on models, thermodynamic arguments, and/or observational trends (Gordon et al. 1992; Trenberth 1998; Meehl et al. 2000). Though these thermodynamic arguments and results are likely robust, such conclusions do not necessarily apply to any one specific region however, because they can be overridden by the influence of atmospheric and oceanic circulation patterns. Thus we need to consider the potential for circulation changes as well. To do this absent firm guidance from models, we refocus the approach for summer on the features from observations that tend to be associated with wetter than normal and drier than normal summers and try to assess reasons for changes in their tendencies.

In the Chesapeake Bay region, wetter than normal summers tend to be associated with a more zonal flow across the United States, as indicated in Fig. 3, along with the passage of more frequent synoptic-scale cyclones. Wet summers in specific locations on the east coast are also related to the incidence of hurricanes, whose projected response to greenhouse forcing is still fairly uncertain (Henderson-Sellers et al. 1998). Dry summers in the Chesapeake are characterized by northwesterly flow with a surface anticyclone producing broadscale subsidence. The synoptic signature for this type of pattern is the omega block structure shown in Fig. 2. Dry summers in the southern Great Plains region also tend to be characterized by broadscale ridging over the continent (omega block). In this synoptic configuration the rain-bearing mesoscale systems tend to develop and track farther to the north. Further, the moisture that is sometimes entrained over the continent from the Gulf of Mexico in wetter years by southward displaced storms is inhibited by the prevailing easterly flow over subtropical portions of the continent in dry years (Trenberth and Guillemot 1996; Mo et al. 1997).

In order to project possible changes in summer precipitation regimes in the regions we selected, one would need to know something about the tendency for blocks of this kind to change their frequency of occurrence or persistence. An increase in frequency of omega blocks could well lead to an increase in droughtiness in the regions. The omega block in the stationary wave pattern in summer is much more strongly dependent on surface interactions and heating than is the stationary wave pattern in winter. Continental surface processes are important in developing and maintaining the block, as are land-sea temperature contrasts. For example, lack of continental soil moisture in spring seems to be related to the tendency for blocks to occur (Namias 1982; 1991; Kunkel 1989). The block may also be an extratropical response to an enhanced Walker circulation and La Niña conditions (Trenberth and Guillemot 1996), though connections between ENSO/La Nina and continental U.S. climate (Ting and Wang 1997) are typically less robust in summer. The likely tendency for La Niña responses to greenhouse forcing is largely unknown at this point.

The set of factors that control the occurrence and persistence of omega blocks are either not well known, or involve processes that occur on small scales, or at the atmosphere-land or atmosphere-ocean boundaries that are difficult to model. Smaller-scale processes and boundary layer interactions also plague the prediction problem in wetter years where moisture sources and outbreaks must be accurately characterized. One can certainly imagine scenarios in which a shift to much wetter or much drier summer conditions might occur in either of the case study regions. Yet there is little firm guidance to base an expectation of one trend or the other at this point, and any such scenarios would be mostly speculation. 


\section{Discussion and conclusions}

The projection of regional climate changes is portrayed here as a challenging and difficult exercise that requires an understanding of the dynamics of the climate system. Climate models embody our best representations of the dynamics. However, their projections are ambiguous and subject to known deficiencies. Some of these deficiencies can be partially "corrected" by approaches such as the present one that allow for interpretation of model outputs. For example, a model's predicted response to greenhouse-forced changes in the tropical Pacific Ocean can be corrected using knowledge of model errors in present-day ENSO teleconnections. The ambiguous and contingent nature of climate model scenarios might be better appreciated when accompanied by qualitative descriptions of the changes in dynamics they entail. This exercise provides some of that description, though not for a specific climate model scenario. Rather, we have tried to span a broader range of scenario space based on tendencies of a selection of dynamical features studied in a range of climate model runs. The scenarios are based on dynamical argumentation, and are only as plausible as our understanding of the general working of the climate system. Though imperfect, the method provides a way to highlight some of the steps in the process of building scenarios and to articulate the underlying rationale.

The three winter scenarios we describe correspond loosely to a local north-south displacement of the stationary wave pattern and reduced baroclinicity, to an increase in the amplitude of the pattern, and to a shift in phase of the pattern. Each of these scenarios yield a different climate type for the regions we studied (some wetter, some drier). The tendencies for changes in the dynamical building blocks (stationary wave pattern, Hadley regime, jet streams, PNA, ENSO, etc.) in winter in response to greenhouse forcing are predominantly in the "ambiguous" category we defined. That is, one can usually give competing arguments (or point to the results of different climate model experiments) that project changes in these features of either sign (increases or decreases in intensity, migration poleward or equatorward, more or less zonality, etc.). The ability to argue for changes either way in these features underscores the highly contingent nature of any one particular scenario. In as much as regional climates depend on these features, this will be true of scenarios generated by any means, including climate models. In principle, one could devise sets of diagnostics to discern whether the climate of a particular region was tending more toward one scenario or another; for example, by monitoring PNA trends (Wallace et al. 1993; Corti et al. 1999) or the Arctic Oscillation (Thompson and Wallace 1998).

Regional climate does seem to display some insensitivity to some dynamical perturbations. For example, as was argued in section $3 \mathrm{~b}(6)$, our study regions are apparently somewhat insensitive to climate changes that lead to remote forcing of the stationary wave pattern. While we have identified many competing mechanisms that influence the general circulation and regional climate, future research needs to quantify the relative importance of each mechanism. More idealized models may be useful to interpret dynamical behavior within GCMs (e.g., Miller and Tegen 1999). Dynamical understanding of climate change will become increasingly important for identifying the more robust and plausible features of regional climate changes as the number of climate forcing scenarios increases, which by itself will complicate direct comparison of different models. With a better understanding of the dynamics it may ultimately be possible to provide approximate ordinal likelihood rankings of the scenarios that have been conceived, and thus to provide a richer set of probabilistic guidance in scenario space.

By attempting to connect results from large-scale dynamical models with the synoptic features used to characterize local weather and climate, some important differences in emphasis are apparent. For example, synopticians point to the critical role for regional climates that meanders of both the subtropical and polar jets can play. Precipitation in both our study regions is influenced by the ability of the subtropical jet (and associated storm systems) to entrain Gulf of Mexico moisture. The precise relationship between the zonal mean jet of dynamical studies and the jet features on synoptic maps is unclear. This suggests a need to analyze GCM responses to greenhouse forcing on daily timescales to elucidate more fully the projected responses of the synoptic jets. The archiving of daily output from climate change simulations also allows investigation of whether the large-scale dynamical controls of the present-day regional climate are maintained under climate change (which we assumed to be the case in the present study). Further, it would facilitate evaluation of model synoptic climatologies against observed data, providing a richer base on which to annotate regional climate scenarios as here. Another issue emerging from the approach is that much of what can be projected about regional climate changes in summer is highly speculative. Though progress is being made in understanding the factors giving rise to summer droughts, the features of interest occur on smaller scales than in winter and the theory underlying them is relatively weak. This leaves little basis to interpret GCM predictions in summer, and emphasizes the critical need for studies of summer circulation.

The synoptic-dynamic approach described here to deconstruct and complement climate model climate scenarios might be applied fruitfully in a variety of regions to help develop understanding of the links between large scales and synoptic scales. By forcing an articulation of the chains of reasoning behind climate scenarios, it should give us a better view of how to improve them. Unlike NWP, climate modeling is not at a point where one can produce accurate quantitative forecasts by interpreting model output appropriately. Yet, because of 
the key role played by uncertainties, the production of climate scenarios stands to benefit from the application of structural methods that can identify critical uncertainties and link them to specific regional outcomes.

Acknowledgments. We thank Jim Titus, Joel Smith, and Michael MacCracken for their thoughtful advice and comments on this project, along with Kevin Trenberth and several anonymous reviewers. Mike Richman provided valuable contributions to the southern Great Plains work described here, as did Paul Kushner for results from the GFDL model. Ray Sterner provided the map used in Fig. 1. This work was supported by the U.S. EPA under subcontract to Stratus Consulting Inc.

“Anyway, don't rely on me to provide a scenario. Anything introduced into this setting decays with alarming speed. A continual fermentation decomposes forms in order to compose others even more complex and ephemeral, and ideas must suffer the same fate."

-Bouvier (1987)

\section{REFERENCES}

Barnett, T., 1999: Comparison of near-surface air temperature variability in 11 coupled global climate models. J. Climate, 12, 511518.

Boer, G., G. Flato, and D. Ramsden, 2000: A transient climate change simulation with greenhouse gas and aerosol forcing: Projected climate to the twenty-first century. Climate Dyn., 16, 427-450.

Bosart, L., C. Lai, and E. Rogers, 1995: Incipient explosive marine cyclogenesis-Coastal development. Tellus, 47, 1-29.

Bouvier, N., 1987: The Scorpion-Fish. Carcenet, 123 pp.

Boyle, J. S., 1998: Evaluation of the annual cycle of precipitation over the United States in GCMs: AMIP simulations. J. Climate, 11, 1041-1055.

Cane, M., 1998: Climate change-A role for the tropical Pacific. Science, 282, p. 59.

— A. Clement, A. Kaplan, Y. Kushnir, D. Pozdnyakov, R. Seager, and S. Zebiak, 1997: Twentieth century sea surface temperature trends. Science, 275, 957-960.

Carnell, R., and C. Senior, 1998: Changes in mid-latitude variability due to increasing greenhouse gases and sulphate aerosols. Climate Dyn., 14, 369-383.

Collins, D., and D. Karoly, 1996: Eddy transports in a greenhouse climate simulation. Aust. Meteor. Mag., 45, 113-122.

Corti, S., F. Molteni, and T. Palmer, 1999: Signature of recent climate change in frequencies of natural atmospheric circulation regimes. Nature, 398, 799-802.

Delworth, T., J. Mahlman, and T. Knutson, 1999: Changes in heat index associated with $\mathrm{CO}_{2}$-induced global warming. Climatic Change, 43, 369-386.

Dole, R., and R. Black, 1990: Life cycles of persistent anomalies. Part II: The development of persistent negative height anomalies over the North Pacific Ocean. Mon. Wea. Rev., 118, 824-846.

Giorgi, F., and L. Mearns, 1991: Approaches to the simulation of regional climate change: A review. Rev. Geophy., 29, 191-216.

Gitelman, A., J. Risbey, R. Kass, and R. Rosen, 1997: Trends in the surface meridional temperature gradient. Geophys. Res. Lett., 24, $1243-1246$.

, — $\longrightarrow$, and $\longrightarrow$ 1999: Sensitivity of a meridional temperature gradient index to latitudinal domain. J. Geophys. Res., 104, $16709-16717$.

Gleick, P. H., 1987: Regional hydrologic consequences of increases in atmospheric $\mathrm{CO}_{2}$ and other trace gases. Climatic Change, 10, $137-161$.

Gordon, H., P. Whetton, A. Pittock, A. Fowler, and M. Haylock, 1992: Simulated changes in daily rainfall intensity due to the enhanced greenhouse-effect: Implications for extreme rainfall events. Climate Dyn., 8, 83-102.

Grotch, S., and M. MacCracken, 1991: The use of general circulation models to predict regional climatic change. J. Climate, 4, 286303.

Hall, N., B. Hoskins, P. Valdes, and C. Senior, 1994: Storm tracks in a high resolution GCM with doubled carbon dioxide. Quart. J. Roy. Meteor. Soc., 120, 1209-1230.

Hansen, J., D. Rind, A. Delgenio, A. Lacis, S. Lebedeff, M. Prather, R. Ruedy, and T. Karl, 1989: Regional greenhouse climate effects. Proc. of the $2 d$ North American Conf. on Preparing for Climate Change, Washington, DC, Climate Institute, 1-18.

Held, I., 1983: Stationary and quasi-stationary eddies in the extratropical troposphere: Theory. Large Scale Dynamical Processes in the Atmosphere, B. Hoskins and R. Pierce, Eds., Academic Press, 127-168.

_ 1993: Large-scale dynamics and global warming. Bull. Amer. Meteor. Soc., 74, 228-241.

__ , and M. Ting, 1990: Orographic versus thermal forcing: The importance of the mean low level wind. J. Atmos. Sci., 47, 495500 .

Henderson-Sellers, A., and Coauthors, 1998: Tropical cyclones and global climate change: A post-IPCC assessment. Bull. Amer. Meteor. Soc., 79, 19-38.

Horel, J., and J. Wallace, 1981: Planetary scale atmospheric phenomena associated with the Southern Oscillation. Mon. Wea. Rev., 109, 813-829.

Hoskins, B., and P. Valdes, 1990: On the existence of storm tracks. J. Atmos. Sci., 47, 1854-1864.

Hou, A., and R. Lindzen, 1992: The influence of concentrated heating on the Hadley circulation. J. Atmos. Sci., 49, 1233-1241.

Houghton, J., Y. Ding, D. Griggs, M. Noguer, P. van der Linden, and D. Xiaosu, Eds., 2001: Climate Change 2001: The Scientific Basis: Contribution of Working Group 1 to the Third Assessment Report of the Intergovernmental Panel on Climate Change. Cambridge University Press, $944 \mathrm{pp}$.

Jeton, A., M. Dettinger, and J. Smith, 1996: Potential effects of climate change on streamflow, eastern and western slopes of the Sierra Nevada, California and Nevada. U.S. Geological Survey Water-Resources Investigations Rep. 95-4260, 60 pp.

Kageyama, M., P. Valdes, G. Ramstein, C. Hewitt, and U. Wyputta, 1999: Northern Hemisphere storm tracks in present day and last glacial maximum climate simulations: A comparison of the European PMIP models. J. Climate, 12, 742-760.

Kunkel, K., 1989: A surface-energy budget view of the 1988 midwestern United States drought. Bound.-Layer Meteor., 48, 217 225 .

Lackmann, G. M., D. Keyser, and L. F. Bosart, 1997: A characteristic life cycle of upper-tropospheric cyclogenetic precursors during the Experiment on Rapidly Intensifying Cyclones over the Atlantic (ERICA). Mon. Wea. Rev., 125, 2729-2758.

Lamb, P. J., 1987: On the development of regional climatic scenarios for policy-oriented climatic-impact assessment. Bull. Amer. Meteor. Soc., 68, 1116-1123.

Lau, K., P. Sheu, and I. Kang, 1994: Multiscale low-frequency modes of circulation in the global atmosphere. J. Atmos. Sci., 51, 11691193

Lau, N., and M. Nath, 1996: The role of the "atmospheric bridge" in linking tropical Pacific ENSO events to extratropical SST anomalies. J. Climate, 9, 2036-2057.

_ - S. Philander, and M. Nath, 1992: Simulation of ENSO-like phenomena with a low-resolution coupled GCM of the global ocean and atmosphere. J. Climate, 5, 284-307.

Leathers, D., B. Yard, and M. Palecki, 1991: The Pacific/North American teleconnection pattern and United States climate. Part I: 
Regional temperature and precipitation anomalies. J. Climate, 4, 517-528.

Lettenmaier, D., and T. Y. Gan, 1990: Hydrologic sensitivities of the Sacramento-San Joaquin river basin, California, to global warming. Water Resour. Res., 26, 69-86.

Lindzen, R., 1994: Climate dynamics and global change. Annu. Rev. Fluid Mech., 26, 353-378.

— heating centered off the equator. J. Atmos. Sci., 45, 2417-2427.

Manabe, S., R. Stouffer, M. Spelman, and K. Bryan, 1991: Transient responses of a coupled ocean-atmosphere model to gradual changes of atmospheric $\mathrm{CO}_{2}$. Part I: Annual mean response. $J$. Climate, 4, 785-818.

Mearns, L., P. Gleick, and S. Schneider, 1990: Climate forecasting. Climate Change and U.S. Water Resources, P. Waggoner, Ed., Wiley, 87-137.

Meehl, G., F. Zwiers, J. Evans, T. Knutson, L. Mearns, and P. Whetton, 2000: Trends in extreme weather and climate events: Issues related to modeling extremes in projections of future climate change. Bull. Amer. Meteor. Soc., 81, 427-436.

Miller, R., and I. Tegen, 1999: Radiative forcing of a tropical direct circulation by soil dust aerosols. J. Atmos. Sci., 56, 2403-2433.

Mo, K. C., J. Paegle, and R. Higgins, 1997: Atmospheric processes associated with summer floods and droughts in the central United States. J. Climate, 10, 3028-3046.

Montroy, D. L., M. B. Richman, and P. J. Lamb, 1998: Observed nonlinearities of monthly teleconnections between tropical Pacific sea surface temperature anomalies and central and eastern North American precipitation. J. Climate, 11, 1812-1835.

Morgan, M., and J. Neilsen-Gammon, 1998: Using tropopause maps to diagnose midlatitude weather systems. Mon. Wea. Rev., 126, $2555-2579$.

Namias, J., 1982: Anatomy of Great Plains protracted heat waves (especially the 1980 U.S. summer drought). Mon. Wea. Rev., 110, 824-838.

_ 1991: Spring and summer 1988 drought over the contiguous United States-Causes and prediction. J. Climate, 4, 54-65.

Nicholls, N., 1999: Cognitive illusions, heuristics, and climate prediction. Bull. Amer. Meteor. Soc., 80, 1385-1397.

Nigam, S., and R. Lindzen, 1989: The sensitivity of stationary waves to variations in the basic state zonal flow. J. Atmos. Sci., 46, $1746-1768$

Palmen, E., and C. Newton, 1969: Atmospheric Circulation Systems. Academic Press, 603 pp.

Peixoto, J., and A. Oort, 1992: Physics of Climate. American Institute of Physics, 520 pp.

Pittock, A. B., and M. Salinger, 1981: Towards regional scenarios for a $\mathrm{CO}_{2}$-warmed earth. Climatic Change, 4, 23-40.

Reader, M., and G. Boer, 1998: The modification of greenhouse gas warming by the direct effect of sulfate aerosols. Climate Dyn., 14, 543-608.

Rind, D., 1998: Latitudinal temperature gradients and climate change. J. Geophys. Res., 103 (D6), 5943-5971.

Risbey, J., and P. Stone, 1996: A case study of the adequacy of GCM simulations for input to regional climate change assessments. $J$. Climate, 9, 1441-1467.

— , P. Kushner, P. Lamb, R. Miller, M. Morgan, M. Richman, G. Roe, and J. Smith, 1999: Generating regional climate scenarios by combining synoptic-climatological guidance and GCM output. U.S. EPA Research Report, 88 pp.

Ropelewski, C., and M. Halpert, 1986: North American precipitation and temperature patterns associated with the El Niño-Southern Oscillation. Mon. Wea. Rev., 114, 2352-2362.

Russell, G., J. Miller, and D. Rind, 1995: A coupled atmosphere- ocean model for transient climate change studies. Atmos.-Ocean, 33, 683-730.

,,,- R. Ruedy, G. Schmidt, and S. Sheth, 2000: Comparison of model and observed regional temperature changes during the past 40 years. J. Geophys. Res., 105, 14 891-14 898.

Sanders, F., 1979: Trends in skill of daily forecasts of temperature and precipitation, 1966-78. Bull. Amer. Meteor. Soc., 60, $763-$ 769.

, 1986: Trends in skill of Boston forecasts made at MIT, 1966 84. Bull. Amer. Meteor. Soc., 67, 170-176.

Schneider, S. H., 1994: Detecting climatic change signals-Are there any fingerprints? Science, 263, 341-347.

Siegmund, P., 1992: Linear simulation of the stationary eddy response of a general circulation model to a doubling of atmospheric $\mathrm{CO}_{2}$. Climate Dyn., 7, 29-37.

Smith, J., and L. Kalkstein, 1995: Introduction. As Climate Changes: International Impacts and Implications, J. Smith and K. Strzepek, Eds., Cambridge University Press, 19-26.

Spetzler, C., and S. von Holstein, 1975: Probability encoding in decision analysis. Manage. Sci., 22, 340-358.

Stephenson, D., and I. Held, 1993: GCM response of northern winter stationary waves and storms tracks to increasing amounts of carbon dioxide. J. Climate, 6, 1859-1870.

Thompson, D., and J. Wallace, 1998: The Arctic Oscillation signature in the wintertime geopotential height and temperature fields. Geophys. Res. Lett., 25, 1297-1300.

Timmermann, A., J. Oberhuber, A. Bacher, M. Esch, M. Latif, and E. Roeckner, 1999: Increased El Niño frequency in a climate model forced by future greenhouse warming. Nature, 398, 694697.

Ting, M., and H. Wang, 1997: Summertime U.S. precipitation variability and its relation to Pacific sea surface temperature. J. Climate, 10, 1853-1873.

Trenberth, K., 1997: Short-term climate variations: Recent accomplishments and issues for future progress. Bull. Amer. Meteor. Soc., 78, 1081-1096.

, 1998: Atmospheric moisture residence times and cycling: Implications for rainfall rates and climate change. Climatic Change, 39, 667-694.

_ by Himalayan orography. J. Atmos. Sci., 45, 2934-2948.

- and C. Guillemot, 1996: Physical processes involved in the 1988 drought and 1993 floods in North America. J. Climate, 9, $1288-1298$.

— lation event: Longest on record. Geophys. Res. Lett., 23, 57-60.

Wallace, J., and D. Gutzler, 1981: Teleconnections in the geopotential height field during the Northern Hemisphere winter. Mon. Wea. Rev., 109, 784-811.

, Y. Zhang, and K.-H. Lau, 1993: Structure and seasonality of interannual and interdecadal variability of the geopotential height and temperature fields in the Northern Hemisphere troposphere. J. Climate, 6, 2063-2082.

Wang, H., and M. Ting, 1999: Seasonal cycle of the climatological stationary waves in the NCEP-NCAR reanalysis. J. Atmos. Sci., 56, 3892-3919.

Wigley, T. M. L., P. D. Jones, and P. M. Kelly, 1986: Empirical climate studies: Warm world scenarios and the detection of a $\mathrm{CO}_{2}$ induced climatic change induced by radiatively active gases. The Greenhouse Effect, Climatic Change, and Ecosystems, B. Bolin et al., Eds., Wiley, 271-322.

Yukimoto, S., M. Endoh, Y. Kitamura, A. Kitoh, T. Motoi, and A. Noda, 2000: ENSO-like interdecadal variability in the Pacific Ocean as simulated in a coupled general circulation model. $J$. Geophys. Res., 105 (C6), 13 945-13 963. 